\title{
Laplace decomposition for solving nonlinear system of fractional order partial differential equations
}

\author{
Hassan Khan ${ }^{1}$ (D, Rasool Shah' ${ }^{1}$ Poom Kumam²,3* Dumitru Baleanu ${ }^{4,5}$ and Muhammad Arif ${ }^{1}$
}

*Correspondence:

poom.kum@kmutt.ac.th

${ }^{2}$ Theoretical and Computational Science (TaCS) Center Department of Mathematics, Faculty of Science, King Mongkuts University of Technology Thonburi (KMUTT), 126 Pracha Uthit Rd., Bang Mod, Thung Khru, 10140 Bangkok, Thailand 3 Department of Medical Research, China Medical University Hospital, China Medical University, 40402 Taichung, Taiwan

Full list of author information is available at the end of the article

\begin{abstract}
In the present article a modified decomposition method is implemented to solve systems of partial differential equations of fractional-order derivatives. The derivatives of fractional-order are expressed in terms of Caputo operator. The validity of the proposed method is analyzed through illustrative examples. The solution graphs have shown a close contact between the exact and LADM solutions. It is observed that the solutions of fractional-order problems converge towards the solution of an integer-order problem, which confirmed the reliability of the suggested technique. Due to better accuracy and straightforward implementation, the extension of the present method can be made to solve other fractional-order problems.
\end{abstract}

Keywords: Caputo operator; Adomian decomposition method; Laplace transformation; Fractional systems of partial differential equations

\section{Introduction}

In the last decade, scientists and engineers have paid much attention towards nonlinear equations, as the nonlinearity exists everywhere in most of the physical problems. The nonlinear partial differential equations of fractional order (FPDEs) are the special case of nonlinear equations that have many applications in science and technology, including chemistry, biology, physics, vibration, acoustic, signals processing, electromagnetic, polymeric materials, and fluid dynamics, super conductivity, optics, and quantum mechanics [1-4]. Due to frequent appearance of FPDEs in different disciplines of engineering and science, the researchers have added a lot of research contribution to both theory of mathematical science and technology [5-9].

In mathematical analysis, the most frequent developing area is fractional-order calculus. In fact, many physical phenomena are dependent on time instant as well as at the earlier history of time are modeled by using fractional-order ordinary differential equations (FODEs), and thus FDEs have attained their importance in many fields of applied sciences. Due to the importance of FDEs, many researchers have made their focus on the analytical solution as well as the numerical solutions of FDEs [10-12]. In this regard numerous techniques have been discussed for the solutions of FPDEs such as homotopy

(c) The Author(s) 2020. This article is licensed under a Creative Commons Attribution 4.0 International License, which permits use sharing, adaptation, distribution and reproduction in any medium or format, as long as you give appropriate credit to the original author(s) and the source, provide a link to the Creative Commons licence, and indicate if changes were made. The images or other third party material in this article are included in the article's Creative Commons licence, unless indicated otherwise in a credit line to the material. If material is not included in the article's Creative Commons licence and your intended use is not permitted by statutory regulation or exceeds the permitted use, you will need to obtain permission directly from the copyright holder. To view a copy of this licence, visit http://creativecommons.org/licenses/by/4.0/. 
analysis method (HAM), homotopy perturbation technique (HPM), Laplace transformation, variational technique with Pade approximation, corrected Fourier series, natural decomposition method [13-16], and fractional complex transformation [17]. The optimal q-HAM is discussed in [18] to solve FPDEs.

Numerical solution of FPDEs has been discussed in [19] efficiently by using Bernoulli wavelets and collocation method. Auxiliary Laplace parameter method has been discussed for the solution of FDEs [20]. Exact solution of Kodomtsev-Petviashvili (KP) equation is obtained in [21] by using simple equation method. Modified variational iteration technique is developed in [2] for the result of nonlinear PDEs. The solution of linear and nonlinear FPDEs has been studied in [22] by using iterative Laplace transform method. Biological nonlinear phenomena like shallow water waves and multicellular biological dynamics can be modeled in terms of nonlinear PDEs of integer order [23]. Numerous FPDEs do not have exact or analytical solution, so numerical methods are used as an alternative. In this regard, numerical solutions of FPDEs have been obtained in [24] by using tau approximation. Discrete HAM is suggested to solve linear and nonlinear FPDEs [2527].

Laplace-Adomian decomposition method (LADM) is one of the effective and straightforward techniques to solve nonlinear FPDEs. LADM possesses the combined behavior of Laplace transformation and Adomian decomposition method (ADM). It is observed that the suggested method requires no predefined declaration size like RK4. LADM requires fewer number of parameters, no discretization and linearization as compared to other analytical techniques [28]. LADM is also compared with ADM to analyze the solution of FPDEs given in [29]. The solution of Kundu-Eckhaus equation is discussed in [30] via LADM. Multistep LADM is implemented to solve FPDEs in [31, 32]. LADM is also used for the solution of fractional Navier-Stokes, Smoke models, and third-order dispersive PDEs [33-35].

In the current study, we implement LADM for the solution of some nonlinear system of FPDEs. The desired degree of accuracy is achieved. The procedure of the suggested technique is very simple and straightforward. The accuracy is calculated in terms of absolute error. The results have shown that the present method has the desired accuracy as compared to other analytical techniques.

\section{Definitions and preliminaries}

Definition 2.1 The Riemann-Liouville definition of fractional integral of a function $g$ with order $\beta \geq 0$ can be expressed as [36,37]

$$
I_{\xi}^{\beta} g(\xi)= \begin{cases}g(\xi) & \text { if } \beta=0 \\ \frac{1}{\Gamma(\beta)} \int_{0}^{\xi}(\xi-v)^{\beta-1} g(v) d v & \text { if } \beta>0\end{cases}
$$

where $\Gamma$ is denoted as

$$
\Gamma(\omega)=\int_{0}^{\infty} e^{-\xi} \xi^{\omega-1} d \xi \quad \omega \in \mathbb{C} .
$$


Definition 2.2 The Caputo definition of fractional derivative of order $\beta$ is described as $[36,37]$

$$
D_{\xi}^{\beta} g(\xi)=\frac{1}{\Gamma(m-\beta)} \int_{0}^{\xi}(\xi-\tau)^{m-\beta-1} g^{(m)}(\tau) d \tau
$$

for $m-1<\beta \leq m, m \in \mathbb{N}, \xi>0, g \in \mathbb{C}_{\tau}, \tau \geq-1$.

Lemma 2.3 If $m-1<\beta \leq m$ with $m \in \mathbb{N}$ and $g \in \mathbb{C}_{\tau}$ with $\tau \geq-1$, then $[36,38]$

$$
\begin{aligned}
& D_{\xi}^{\beta} I_{\xi}^{\beta} g(\xi)=g(\xi), \\
& I^{\beta} \xi^{\lambda}=\frac{\Gamma(\lambda+1)}{\Gamma(\beta+\lambda+1)} \xi^{\beta+\lambda}, \quad \beta>0, \lambda>-1, \xi>0, \\
& D_{\xi}^{\beta} I_{\xi}^{\beta} g(\xi)=g(\xi)-\sum_{k=0}^{m} g^{(k)}\left(0^{+}\right) \frac{\xi^{k}}{k !} \quad \text { for } \xi>0 .
\end{aligned}
$$

Definition 2.4 The Laplace transform $G(s)$ of $g(\tau)$ is expressed as [30]

$$
G(s)=\mathcal{L}[g(\tau)]=\int_{0}^{\infty} e^{-s \tau} g(t) d t
$$

Definition 2.5 The Laplace transform of fractional derivative is [30]

$$
\mathcal{L}\left(D_{\tau}^{\beta} g(\tau)\right)=s^{\beta} G(s)-\sum_{k=0}^{m-1} s^{\beta-1-k} g^{(k)}(0), \quad m-1<\beta<m .
$$

\section{LADM idea for FPDEs}

Consider the following FPDE [30]:

$$
D^{\beta} u(\xi, \tau)+L u(\xi, \tau)+N u(\xi, \tau)=q(\xi, \tau), \quad \xi, \tau \geq 0, m-1<\beta \leq m .
$$

The fractional derivative in equation (1) is expressed in the Caputo sense. The linear and nonlinear terms are denoted by $L$ and $N$ respectively, and $q(\xi, \tau)$ is the sources term with the initial condition

$$
u(\xi, 0)=f(\xi) .
$$

Applying the Laplace transform on both sides of equation (1), we get [30]

$$
\begin{aligned}
& s^{\beta} \mathcal{L}[u(\xi, \tau)]-s^{\beta-1} u(\xi, 0)=\mathcal{L}[q(\xi, \tau)]-\mathcal{L}[L u(\xi, \tau)+N u(\xi, \tau)] \\
& \mathcal{L}[u(\xi, \tau)]=\frac{k(\xi)}{s}-\frac{1}{s^{\beta}} \mathcal{L}[L u(\xi, \tau)+N u(\xi, \tau)+q(\xi, \tau)] .
\end{aligned}
$$

The ADM solution is

$$
u(\xi, \tau)=\sum_{j=0}^{\infty} u_{j}(\xi, \tau)
$$


The nonlinear term in the problem is expressed as

$$
N u(\xi, \tau)=\sum_{j=0}^{\infty} A_{j}
$$

where

$$
A_{j}=\frac{1}{j !}\left[\frac{d^{j}}{d \lambda}\left[N \sum_{j=0}^{\infty}\left(\lambda^{j} u_{j}\right)\right]\right]_{\lambda=0}, \quad j=0,1,2, \ldots,
$$

are called Adomian polynomials.

Substituting equations (4) and (5) in equation (3), we get

$$
\mathcal{L}\left[\sum_{j=0}^{\infty} u(\xi, \tau)\right]=\frac{f(x)}{s}-\frac{1}{s^{\beta}} \mathcal{L}\left[L \sum_{j=0}^{\infty} u_{j}(\xi, \tau)+\sum_{j=0}^{\infty} A_{j}+q(\xi, \tau)\right] .
$$

Applying the decomposition method, we get

$$
\mathcal{L}\left[u_{0}(\xi, \tau)\right]=\frac{f(\xi)}{s}
$$

and

$$
\mathcal{L}\left[u_{j+1}(\xi, \tau)\right]=-\frac{1}{s^{\beta}} \mathcal{L}\left[L u_{j}(\xi, \tau)+A_{j}+q(\xi, \tau)\right], \quad j \geq 1
$$

Using the inverse transform to equations (7) and (8), we have [30]

$$
\begin{aligned}
& u_{0}(\xi, \tau)=f(\xi) \\
& u_{j+1}(\xi, \tau)=-\mathcal{L}^{-1}\left[\frac{1}{s^{\beta}} \mathcal{L}\left[L u_{j}(\xi, \tau)+A_{j}\right]\right] .
\end{aligned}
$$

\section{Theorem}

Here, we study the convergence analysis in the same manner as in [39] of the LADM applied to PDEs. Let us consider the Hilbert space $\mathrm{H}$ which may define by $H=L^{2}((\alpha, \beta) X[0$, $T]$ ) the set of applications

$$
u:(\alpha, \beta) X[0, T] \rightarrow \quad \text { with } \int_{(\alpha, \beta) X[0, T]} u^{2}(\xi, s) d s d \theta<+\infty .
$$

Now we consider the PDEs in the light of the above assumptions. Let us denote

$$
L(u)=\frac{\partial^{\gamma} u}{\partial \tau^{\gamma}},
$$

then the fractional-order of PDEs becomes, in an operator form,

$$
L(u)=\varphi \frac{\partial v(\xi, \tau)}{\partial \xi}-w \frac{\partial v(\zeta, \tau)}{\partial \zeta}
$$

The LADM reaches convergence if the following two hypotheses are satisfied: 
(H1) $(L(u)-L(v), u-v) \geq k\|u-v\|^{2} ; k>0, \forall u, v \in H$.

(H2) Whatever may be $M>0$, there exists a constant $C(M)>0$ such that, for $u, v \in H$ with $\|u\| \leq M,\|v\| \leq M$, we have $(L(u)-L(v), u-v) \leq C(M)\|u-v\|\|w\|$ for every $w \epsilon H$.

Example 1 The system of fractional-order PDEs in [40]

$$
\begin{aligned}
& \frac{\partial^{\beta} u}{\partial \tau^{\beta}}-v \frac{\partial u}{\partial \xi}-\frac{\partial v}{\partial \tau} \frac{\partial u}{\partial \eta}=1-\xi+\eta+\tau, \\
& \frac{\partial^{\beta} v}{\partial \tau^{\beta}}-u \frac{\partial v}{\partial \xi}+\frac{\partial u}{\partial \tau} \frac{\partial v}{\partial \eta}=1-\xi-\eta-\tau, \quad 0<\beta \leq 1,
\end{aligned}
$$

with the initial condition

$$
u(\xi, \eta, 0)=\xi+\eta-1, \quad v(\xi, \eta, 0)=\xi-\eta+1 .
$$

Taking the Laplace transform of equation (10), we have

$$
\begin{aligned}
& \mathcal{L}\left[\frac{\partial^{\beta} u}{\partial \tau^{\beta}}\right]=\mathcal{L}\left[v \frac{\partial u}{\partial \xi}+\frac{\partial v}{\partial \tau} \frac{\partial u}{\partial \eta}+1-\xi+\eta+\tau\right], \\
& \mathcal{L}\left[\frac{\partial^{\beta} v}{\partial \tau^{\beta}}\right]=\mathcal{L}\left[u \frac{\partial v}{\partial \xi}-\frac{\partial u}{\partial \tau} \frac{\partial v}{\partial \eta}+1-\xi-\eta-\tau\right], \\
& s^{\beta} \mathcal{L}[u(\xi, \eta, \tau)]-s^{\beta-1}[u(\xi, \eta, 0)]=\mathcal{L}\left[v \frac{\partial u}{\partial \xi}+\frac{\partial v}{\partial \tau} \frac{\partial u}{\partial \eta}+1-\xi+\eta+\tau\right], \\
& s^{\beta} \mathcal{L}[v(\xi, \eta, \tau)]-s^{\beta-1}[v(\xi, \eta, 0)]=\mathcal{L}\left[u \frac{\partial v}{\partial \xi}-\frac{\partial u}{\partial \tau} \frac{\partial v}{\partial \eta}+1-\xi-\eta-\tau\right] .
\end{aligned}
$$

Using the inverse transformation, we get

$$
\begin{aligned}
& u(\xi, \eta, \tau)=\mathcal{L}^{-1}\left[\frac{u(\xi, \eta, 0)}{s}+\frac{1}{s^{\beta}} \mathcal{L}\left[\nu \frac{\partial u}{\partial \xi}+\frac{\partial v}{\partial \tau} \frac{\partial u}{\partial \eta}+1-\xi+\eta+\tau\right]\right] \\
& v(\xi, \eta, \tau)=\mathcal{L}^{-1}\left[\frac{\nu(\xi, \eta, 0)}{s}+\frac{1}{s^{\beta}} \mathcal{L}\left[u \frac{\partial v}{\partial \xi}-\frac{\partial u}{\partial \tau} \frac{\partial v}{\partial \eta}+1-\xi-\eta-\tau\right]\right] \\
& u(\xi, \eta, \tau)=\xi+\eta-1+\mathcal{L}^{-1}\left[\frac{1}{s^{\beta}} \mathcal{L}\left[v \frac{\partial u}{\partial \xi}+\frac{\partial v}{\partial \tau} \frac{\partial u}{\partial \eta}+1-\xi+\eta+\tau\right]\right] \\
& v(\xi, \eta, \tau)=\xi-\eta+1+\mathcal{L}^{-1}\left[\frac{1}{s^{\beta}} \mathcal{L}\left[u \frac{\partial v}{\partial \xi}-\frac{\partial u}{\partial \tau} \frac{\partial v}{\partial \eta}+1-\xi-\eta-\tau\right]\right]
\end{aligned}
$$

Using the ADM procedure, we get

$$
\begin{aligned}
& \sum_{j=0}^{\infty} u_{j}(\xi, \eta, \tau)=\xi+\eta-1+\mathcal{L}^{-1}\left[\frac{1}{s^{\beta}} \mathcal{L}\left[\sum_{j=0}^{\infty} A_{j}(v, u)+\sum_{j=0}^{\infty} B_{j}(v, u)+1-\xi+\eta+\tau\right]\right], \\
& \sum_{j=0}^{\infty} v_{j}(\xi, \eta, \tau)=\xi-\eta+1+\mathcal{L}^{-1}\left[\frac{1}{s^{\beta}} \mathcal{L}\left[\sum_{j=0}^{\infty} C_{j}(u, v)-\sum_{j=0}^{\infty} D_{j}(u, v)+1-\xi-\eta-\tau\right]\right]
\end{aligned}
$$


where Adomian polynomial components $A_{j}(v, u), B_{j}(v, u), C_{j}(u, v)$, and $D_{j}(u, v)$ are given as follows:

$$
\begin{aligned}
& A_{0}(v, u)=v_{0} \frac{\partial u_{0}}{\partial \xi}, \\
& A_{1}(v, u)=v_{0} \frac{\partial u_{1}}{\partial \xi}+v_{1} \frac{\partial u_{0}}{\partial \xi}, \\
& A_{2}(v, u)=v_{0} \frac{\partial u_{2}}{\partial \xi}+v_{1} \frac{\partial u_{1}}{\partial \xi}+v_{2} \frac{\partial u_{0}}{\partial \xi}, \\
& B_{0}(v, u)=\frac{\partial v_{0}}{\partial \tau} \frac{\partial u_{0}}{\partial \eta}, \\
& B_{1}(v, u)=\frac{\partial v_{0}}{\partial \tau} \frac{\partial u_{1}}{\partial \eta}+\frac{\partial v_{1}}{\partial \tau} \frac{\partial u_{0}}{\partial \eta}, \\
& B_{2}(v, u)=\frac{\partial v_{0}}{\partial \tau} \frac{\partial u_{2}}{\partial \eta}+\frac{\partial v_{1}}{\partial \tau} \frac{\partial u_{1}}{\partial \eta}+\frac{\partial v_{2}}{\partial \tau} \frac{\partial u_{0}}{\partial \eta}, \\
& C_{0}(u, v)=u_{0} \frac{\partial v_{0}}{\partial \xi}, \\
& C_{1}(u, v)=u_{0} \frac{\partial v_{1}}{\partial \xi}+u_{1} \frac{\partial v_{0}}{\partial \xi}, \\
& C_{2}(u, v)=u_{0} \frac{\partial v_{2}}{\partial \xi}+u_{1} \frac{\partial v_{1}}{\partial \xi}+u_{2} \frac{\partial v_{0}}{\partial \xi}, \\
& D_{0}(u, v)=\frac{\partial u_{0}}{\partial \tau} \frac{\partial v_{0}}{\partial \eta}, \\
& D_{1}(u, v)=\frac{\partial u_{0}}{\partial \tau} \frac{\partial v_{1}}{\partial \eta}+\frac{\partial u_{1}}{\partial \tau} \frac{\partial v_{0}}{\partial \eta}, \\
& D_{2}(u, v)=\frac{\partial u_{0}}{\partial \tau} \frac{\partial v_{2}}{\partial \eta}+\frac{\partial u_{1}}{\partial \tau} \frac{\partial v_{1}}{\partial \eta}+\frac{\partial u_{2}}{\partial \tau} \frac{\partial v_{0}}{\partial \eta}, \\
& u_{0}(\xi, \eta, \tau)=\xi+\eta-1, \\
& \nu_{0}(\xi, \eta, \tau)=\xi-\eta+1, \\
& u_{j+1}(\xi, \eta, \tau)=\mathcal{L}^{-1}\left[\frac{1}{s^{\beta}} \mathcal{L}\left\{\sum_{j=0}^{\infty} A_{j}(v, u)+\sum_{j=0}^{\infty} B_{j}(v, u)+1-\xi+\eta+\tau\right\}\right], \\
& v_{j+1}(\xi, \eta, \tau)=\mathcal{L}^{-1}\left[\frac{1}{s^{\beta}} \mathcal{L}\left\{\sum_{j=0}^{\infty} C_{j}(u, v)-\sum_{j=0}^{\infty} D_{j}(u, v)+1-\xi-\eta-\tau\right\}\right]
\end{aligned}
$$

for $j=0,1,2, \ldots$

$$
\begin{aligned}
& u_{1}(\xi, \eta, \tau)=\mathcal{L}^{-1}\left[\frac{1}{s^{\beta}} \mathcal{L}\left\{v_{0} \frac{\partial u_{0}}{\partial \xi}+\frac{\partial v_{0}}{\partial \tau} \frac{\partial u_{0}}{\partial \eta}+1-\xi+\eta+\tau\right\}\right] \\
& u_{1}(\xi, \eta, \tau)=\mathcal{L}^{-1}\left[\frac{2}{s^{\beta+1}}+\frac{1}{s^{\beta+2}}\right]=\frac{2 \tau^{\beta}}{\Gamma(\beta+1)}+\frac{\tau^{\beta+1}}{\Gamma(\beta+2)}, \\
& v_{1}(\xi, \eta, \tau)=\mathcal{L}^{-1}\left[\frac{1}{s^{\beta}} \mathcal{L}\left\{u_{0} \frac{\partial v_{0}}{\partial \xi}-\frac{\partial u_{0}}{\partial \tau} \frac{\partial v_{0}}{\partial \eta}+1-\xi-\eta-\tau\right\}\right] \\
& v_{1}(\xi, \eta, \tau)=\mathcal{L}^{-1}\left[\frac{-1}{s^{\beta+2}}\right]=\frac{-\tau^{\beta+1}}{\Gamma(\beta+2)} .
\end{aligned}
$$


The subsequent terms are

$u_{2}(\xi, \eta, \tau)$

$$
\begin{aligned}
& =\mathcal{L}^{-1}\left[\frac{1}{s^{\beta}} \mathcal{L}\left\{v_{0} \frac{\partial u_{1}}{\partial \xi}+v_{1} \frac{\partial u_{0}}{\partial \xi}+\frac{\partial v_{0}}{\partial \tau} \frac{\partial u_{1}}{\partial \eta}+\frac{\partial v_{1}}{\partial \tau} \frac{\partial u_{0}}{\partial \eta}\right\}\right] \\
& =\frac{-\tau^{2 \beta+1}}{\Gamma(2 \beta+2)}-\frac{\tau^{2 \beta}}{\Gamma(\beta+2)},
\end{aligned}
$$

$v_{2}(\xi, \eta, \tau)$

$$
\begin{aligned}
& =\mathcal{L}^{-1}\left[\frac{1}{s^{\beta}} \mathcal{L}\left\{u_{0} \frac{\partial v_{1}}{\partial \xi}+u_{1} \frac{\partial v_{0}}{\partial \xi}-\frac{\partial u_{0}}{\partial \tau} \frac{\partial v_{1}}{\partial \eta}-\frac{\partial u_{1}}{\partial \tau} \frac{\partial v_{0}}{\partial \eta}\right\}\right] \\
& =\left(\frac{2 \Gamma(\beta+2)-(\beta+1) \Gamma(\beta+1)}{\Gamma(2 \beta+1) \Gamma(\beta+2)}\right) \tau^{2 \beta}+\frac{\tau^{2 \beta+1}}{\Gamma(2 \beta+2)}-\frac{2 \beta \Gamma(\beta) \tau^{2 \beta-1}}{\Gamma(\beta+1) \Gamma(2 \beta)},
\end{aligned}
$$

$u_{3}(\xi, \eta, \tau)$

$$
\begin{aligned}
= & \mathcal{L}^{-1}\left[\frac{1}{s^{\beta}} \mathcal{L}\left\{v_{0} \frac{\partial u_{2}}{\partial \xi}+v_{1} \frac{\partial u_{1}}{\partial \xi}+v_{2} \frac{\partial u_{0}}{\partial \xi}+\frac{\partial v_{0}}{\partial \tau} \frac{\partial u_{2}}{\partial \eta}+\frac{\partial v_{1}}{\partial \tau} \frac{\partial u_{1}}{\partial \eta}+\frac{\partial v_{2}}{\partial \tau} \frac{\partial u_{0}}{\partial \eta}\right\}\right] \\
= & \frac{\tau^{3 \beta+1}}{\Gamma(3 \beta+2)}+\left(\frac{2 \Gamma(\beta+2)-(\beta+1) \Gamma(\beta+1)}{\Gamma(3 \beta+1) \Gamma(\beta+2)}\right) \tau^{3 \beta}+\frac{\tau^{3 \beta}}{\Gamma(2 \beta+2)} \\
& -\frac{2 \beta \Gamma(\beta) \tau^{3 \beta-1}}{\Gamma(\beta+1) \Gamma(3 \beta)}+\left(\frac{(2 \Gamma(\beta+2)-(\beta+1) \Gamma(\beta+1)) 2 \beta \Gamma(\beta)}{\Gamma(3 \beta) \Gamma(2 \beta+1) \Gamma(\beta+2)}\right) \tau^{3 \beta-1} \\
& -\left(\frac{2 \beta \Gamma(\beta)}{\Gamma(2 \beta) \Gamma(\beta+1)}\right) \tau^{3 \beta-2}
\end{aligned}
$$

$\nu_{3}(\xi, \eta, \tau)$

$$
\begin{aligned}
& =\mathcal{L}^{-1}\left[\frac{1}{s^{\beta}} \mathcal{L}\left\{u_{0} \frac{\partial v_{2}}{\partial \xi}+u_{1} \frac{\partial v_{1}}{\partial \xi}+u_{2} \frac{\partial v_{0}}{\partial \xi}-\frac{\partial u_{0}}{\partial \tau} \frac{\partial v_{2}}{\partial \eta}-\frac{\partial u_{1}}{\partial \tau} \frac{\partial v_{1}}{\partial \eta}-\frac{\partial u_{2}}{\partial \tau} \frac{\partial v_{0}}{\partial \eta}\right\}\right] \\
& =\frac{-\tau^{3 \beta+1}}{\Gamma(3 \beta+2)}+\left(\frac{(\beta+1) \Gamma(\beta+1) 2 \beta \Gamma(2 \beta)}{\Gamma(3 \beta) \Gamma(\beta+2) \Gamma(\beta+2)}\right) \tau^{3 \beta-1} .
\end{aligned}
$$

The LADM solution for Example 1 is

$$
\begin{aligned}
u(\xi, \eta, \tau)= & u_{0}(\xi, \eta, \tau)+u_{1}(\xi, \eta, \tau)+u_{2}(\xi, \eta, \tau)+u_{3}(\xi, \eta, \tau)+\cdots, \\
v(\xi, \eta, \tau)= & v_{0}(\xi, \eta, \tau)+v_{1}(\xi, \eta, \tau)+v_{2}(\xi, \eta, \tau)+v_{3}(\xi, \eta, \tau)+\cdots, \\
u(\xi, \eta, \tau)= & \xi+\eta-1+\frac{2 \tau^{\beta}}{\Gamma(\beta+1)}+\frac{\tau^{\beta+1}}{\Gamma(\beta+2)}-\frac{\tau^{2 \beta+1}}{\Gamma(2 \beta+2)}-\frac{\tau^{2 \beta}}{\Gamma(2 \beta+1)} \\
& +\frac{\tau^{3 \beta+1}}{\Gamma(3 \beta+2)}+\left(\frac{2 \Gamma(\beta+2)-(\beta+1) \Gamma(\beta+1)}{\Gamma(3 \beta+1) \Gamma(\beta+2)}\right) \tau^{3 \beta}+\frac{\tau^{3 \beta}}{\Gamma(2 \beta+2)} \\
& -\frac{2 \beta \Gamma(\beta) \tau^{3 \beta-1}}{\Gamma(\beta+1) \Gamma(3 \beta)}+\left(\frac{(2 \Gamma(\beta+2)-(\beta+1) \Gamma(\beta+1)) 2 \beta \Gamma(\beta)}{\Gamma(3 \beta) \Gamma(2 \beta+1) \Gamma(\beta+2)}\right) \tau^{3 \beta-1} \\
& -\left(\frac{2 \beta \Gamma(\beta)}{\Gamma(2 \beta) \Gamma(\beta+1)}\right) \tau^{3 \beta-2}+\cdots, \\
v(\xi, \eta, \tau)= & \xi-\eta+1-\frac{\tau^{\beta+1}}{\Gamma(\beta+2)}+\left(\frac{2 \Gamma(\beta+2)-(\beta+1) \Gamma(\beta+1)}{\Gamma(2 \beta+1) \Gamma(\beta+2)}\right) \tau^{2 \beta}
\end{aligned}
$$



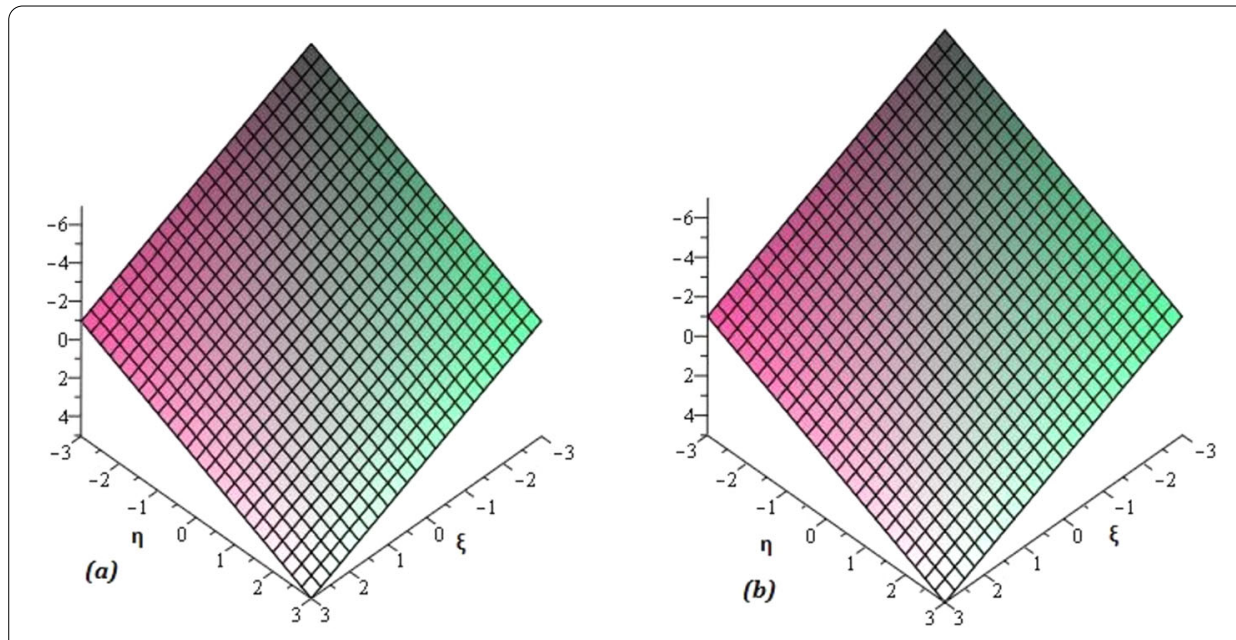

Figure 1 The (a) exact and (b) LADM solutions graph of Example 1 of $u$ at $\beta=1$ and $\tau=0.5$
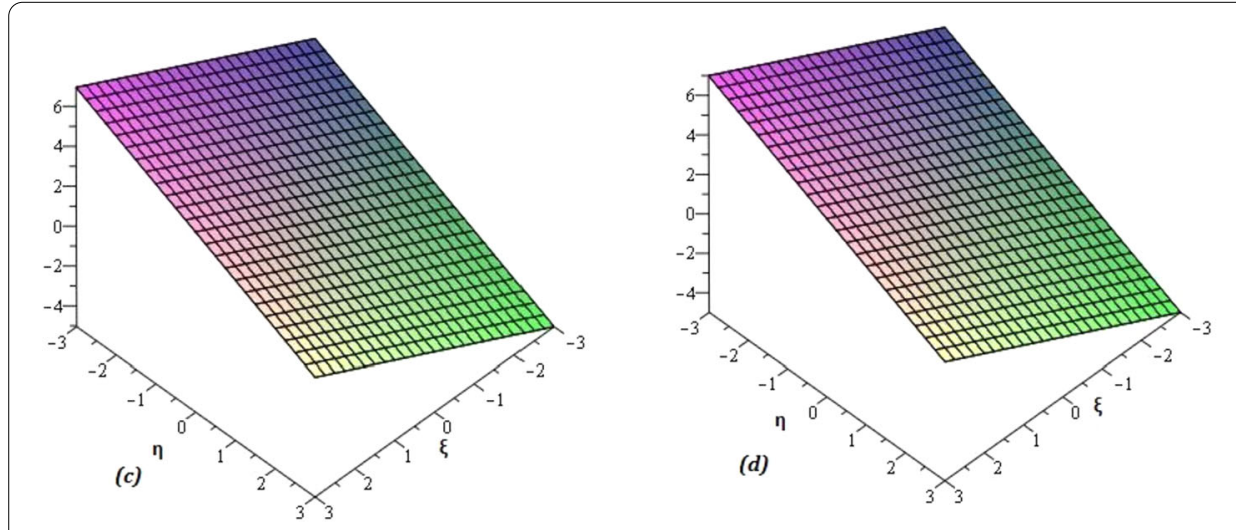

Figure 2 The (c) exact and (d) LADM solution graph of Example 1 of $v$ at $\beta=1$ and $\tau=0.5$

$$
\begin{aligned}
& +\frac{\tau^{2 \beta+1}}{\Gamma(2 \beta+2)}-\frac{2 \beta \Gamma(\beta) \tau^{2 \beta-1}}{\Gamma(\beta+1) \Gamma(2 \beta)}-\frac{\tau^{3 \beta+1}}{\Gamma(3 \beta+2)} \\
& +\left(\frac{(\beta+1) \Gamma(\beta+1) 2 \beta \Gamma(2 \beta)}{\Gamma(3 \beta) \Gamma(\beta+2) \Gamma(\beta+2)}\right) \tau^{3 \beta-1}+\cdots
\end{aligned}
$$

When $\beta=1$, then LADM solution is

$$
\begin{aligned}
& u(\xi, \eta, \tau)=\xi+\eta+\tau-1, \\
& v(\xi, \eta, \tau)=\xi-\eta-\tau+1 .
\end{aligned}
$$

Example 2 The system of fractional-order PDEs in [40]

$$
\begin{aligned}
& \frac{\partial^{\beta} u}{\partial \xi^{\beta}}-v \frac{\partial u}{\partial \tau}+u \frac{\partial v}{\partial \tau}=-1+e^{\xi} \sin \tau, \\
& \frac{\partial^{\beta} v}{\partial \xi^{\beta}}+\frac{\partial u}{\partial \tau} \frac{\partial v}{\partial \xi}-\frac{\partial v}{\partial \tau} \frac{\partial u}{\partial \xi}=-1-e^{-\xi} \sin \tau, \quad 0<\beta \leq 1,
\end{aligned}
$$



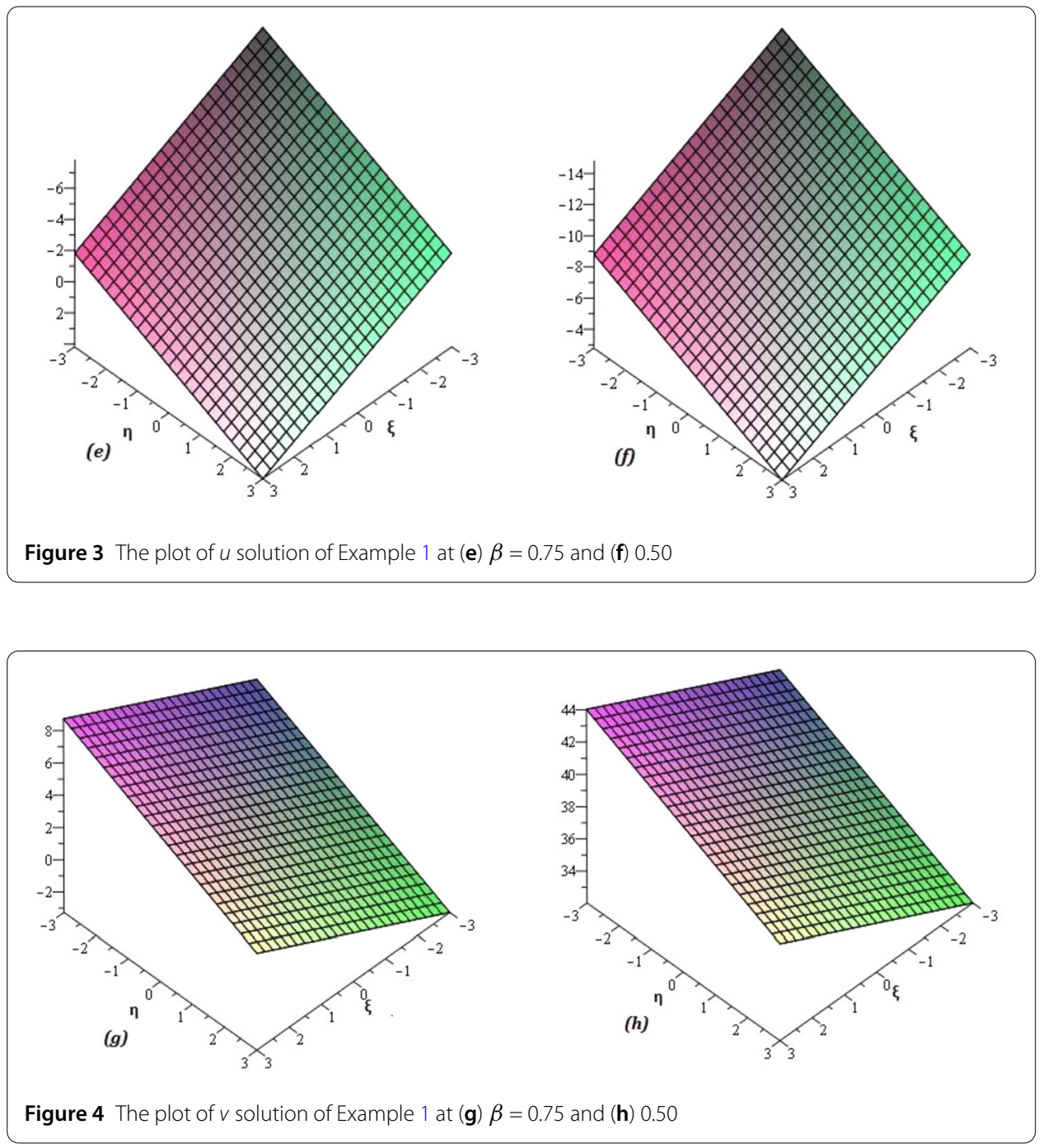

Table 1 LADM solutions of Example 1 at different fractional order $\beta$ and its corresponding absolute error (A.E) of $u$ at $\tau=0.5$

\begin{tabular}{llllll}
\hline$\xi$ & $\eta$ & $\mathrm{A} . \mathrm{E}(\beta=0.55)$ & $\mathrm{A} . \mathrm{E}(\beta=0.75)$ & $\mathrm{A} . \mathrm{E}[\beta=1(j=5)]$ & $\mathrm{A.E}[\beta=1(j=6)]$ \\
\hline 0.1 & 0.1 & $1.639 \mathrm{E}-02$ & $5.828 \mathrm{E}-03$ & $1.000 \mathrm{E}-03$ & $2.000 \mathrm{E}-04$ \\
0.2 & 0.2 & $3.278 \mathrm{E}-02$ & $1.165 \mathrm{E}-02$ & $2.000 \mathrm{E}-03$ & $4.000 \mathrm{E}-04$ \\
0.3 & 0.3 & $4.917 \mathrm{E}-02$ & $1.748 \mathrm{E}-02$ & $3.000 \mathrm{E}-03$ & $6.000 \mathrm{E}-04$ \\
0.4 & 0.4 & $6.556 \mathrm{E}-02$ & $2.331 \mathrm{E}-02$ & $4.000 \mathrm{E}-03$ & $8.000 \mathrm{E}-04$ \\
0.5 & 0.5 & $8.196 \mathrm{E}-02$ & $2.914 \mathrm{E}-02$ & $5.000 \mathrm{E}-03$ & $1.000 \mathrm{E}-03$ \\
\hline
\end{tabular}

with the initial conditions

$$
u(0, \tau)=\sin \tau, \quad v(0, \tau)=\cos \tau .
$$

Taking the Laplace transform of (16), we have

$$
\mathcal{L}\left[\frac{\partial^{\beta} u}{\partial \xi^{\beta}}\right]=\mathcal{L}\left[\nu \frac{\partial u}{\partial \tau}-u \frac{\partial v}{\partial \tau}-1+e^{\xi} \sin \tau\right]
$$


Table 2 LADM solutions of Example 1 at different fractional order $\beta$ and its corresponding absolute error (A.E) of $v$ at $\tau=0.5$

\begin{tabular}{llllll}
\hline$\xi$ & $\eta$ & A.E $(\beta=0.55)$ & A.E $(\beta=0.75)$ & A.E $[\beta=1(j=5)]$ & A.E $[\beta=1(j=6)]$ \\
\hline 0.1 & 0.1 & $1.484 \mathrm{E}-02$ & $5.308 \mathrm{E}-03$ & $9.949 \mathrm{E}-04$ & $9.994 \mathrm{E}-05$ \\
0.2 & 0.2 & $2.969 \mathrm{E}-02$ & $1.061 \mathrm{E}-02$ & $1.989 \mathrm{E}-03$ & $1.998 \mathrm{E}-04$ \\
0.3 & 0.3 & $4.454 \mathrm{E}-02$ & $1.592 \mathrm{E}-02$ & $2.984 \mathrm{E}-03$ & $2.998 \mathrm{E}-04$ \\
0.4 & 0.4 & $5.938 \mathrm{E}-02$ & $2.123 \mathrm{E}-02$ & $3.979 \mathrm{E}-03$ & $3.997 \mathrm{E}-04$ \\
0.5 & 0.5 & $7.423 \mathrm{E}-02$ & $2.654 \mathrm{E}-02$ & $4.974 \mathrm{E}-03$ & $4.997 \mathrm{E}-04$ \\
\hline
\end{tabular}

$$
\begin{aligned}
& \mathcal{L}\left[\frac{\partial^{\beta} v}{\partial \xi^{\beta}}\right]=\mathcal{L}\left[-\frac{\partial u}{\partial \tau} \frac{\partial v}{\partial \xi}-\frac{\partial v}{\partial \tau} \frac{\partial u}{\partial \xi}-1-e^{-\xi} \sin \tau\right], \\
& s^{\beta} \mathcal{L}[u(\xi, \tau)]-s^{\beta-1}[u(0, \tau)]=\mathcal{L}\left[v \frac{\partial u}{\partial \tau}-u \frac{\partial v}{\partial \tau}-1+e^{\xi} \sin \tau\right], \\
& s^{\beta} \mathcal{L}[v(\xi, \tau)]-s^{\beta-1}[v(0, \tau)]=\mathcal{L}\left[-\frac{\partial u}{\partial \tau} \frac{\partial v}{\partial \xi}-\frac{\partial v}{\partial \tau} \frac{\partial u}{\partial \xi}-1-e^{-\xi} \sin \tau\right] .
\end{aligned}
$$

Using the inverse transformation, we obtain

$$
\begin{aligned}
& u(\xi, \tau)=\mathcal{L}^{-1}\left[\frac{u(0, \tau)}{s}+\frac{1}{s^{\beta}} \mathcal{L}\left\{v \frac{\partial u}{\partial \tau}-u \frac{\partial v}{\partial \tau}-1+e^{\xi} \sin \tau\right\}\right] \\
& \nu(\xi, \tau)=\mathcal{L}^{-1}\left[\frac{\nu(0, \tau)}{s}+\frac{1}{s^{\beta}} \mathcal{L}\left\{-\frac{\partial u}{\partial \tau} \frac{\partial v}{\partial \xi}-\frac{\partial v}{\partial \tau} \frac{\partial u}{\partial \xi}-1-e^{-\xi} \sin \tau\right\}\right] .
\end{aligned}
$$

Using the ADM procedure, we get

$$
\begin{aligned}
& u_{0}(\xi, \tau)=\mathcal{L}^{-1}\left[\frac{u(0, \tau)}{s}\right]=\mathcal{L}^{-1}\left[\frac{\sin \tau}{s}\right]=\sin \tau, \\
& v_{0}(\xi, \tau)=\mathcal{L}^{-1}\left[\frac{v(0, \tau)}{s}\right]=\mathcal{L}^{-1}\left[\frac{\cos \tau}{s}\right]=\cos \tau, \\
& u_{j+1}(\xi, \tau)=\mathcal{L}^{-1}\left[\frac{1}{s^{\beta}} \mathcal{L}\left\{v_{j} \frac{\partial u_{j}}{\partial \tau}-u_{j} \frac{\partial v_{j}}{\partial \tau}-1+e^{\xi} \sin \tau\right\}\right], \\
& v_{j+1}(\xi, \tau)=\mathcal{L}^{-1}\left[\frac{1}{s^{\beta}} \mathcal{L}\left\{-\frac{\partial u_{j}}{\partial \tau} \frac{\partial v_{j}}{\partial \xi}-\frac{\partial v_{j}}{\partial \tau} \frac{\partial u_{j}}{\partial \xi}-1-e^{-\xi} \sin \tau\right\}\right], \\
& j=0,1,2, \ldots
\end{aligned}
$$

for $j=0$

$$
\begin{aligned}
& u_{1}(\xi, \tau)=\mathcal{L}^{-1}\left[\frac{1}{s^{\beta}} \mathcal{L}\left\{v_{0} \frac{\partial u_{0}}{\partial \tau}-u_{0} \frac{\partial v_{0}}{\partial \tau}-1+e^{\xi} \sin \tau\right\}\right], \\
& u_{1}(\xi, \tau)=\mathcal{L}^{-1}\left[\frac{\sin \tau}{s^{\beta}(s-1)}\right]=\sin \tau \xi^{\beta} \sum_{k=0}^{\infty} \frac{\xi^{k}}{\Gamma(k+\beta+1)}, \\
& v_{1}(\xi, \tau)=\mathcal{L}^{-1}\left[\frac{1}{s^{\beta}} \mathcal{L}\left\{-\frac{\partial u_{0}}{\partial \tau} \frac{\partial v_{0}}{\partial \xi}-\frac{\partial v_{0}}{\partial \tau} \frac{\partial u_{0}}{\partial \xi}-1-e^{-\xi} \sin \tau\right\}\right], \\
& v_{1}(\xi, \tau)=\mathcal{L}^{-1}\left[\frac{-1}{s^{\beta+1}}+\frac{\cos \tau}{s^{\beta}(s+1)}\right]=\frac{-\tau^{\beta}}{\Gamma(\beta+1)}-\cos \tau \xi^{\beta} \sum_{k=0}^{\infty} \frac{-\xi^{k}}{\Gamma(k+\beta+1)},
\end{aligned}
$$



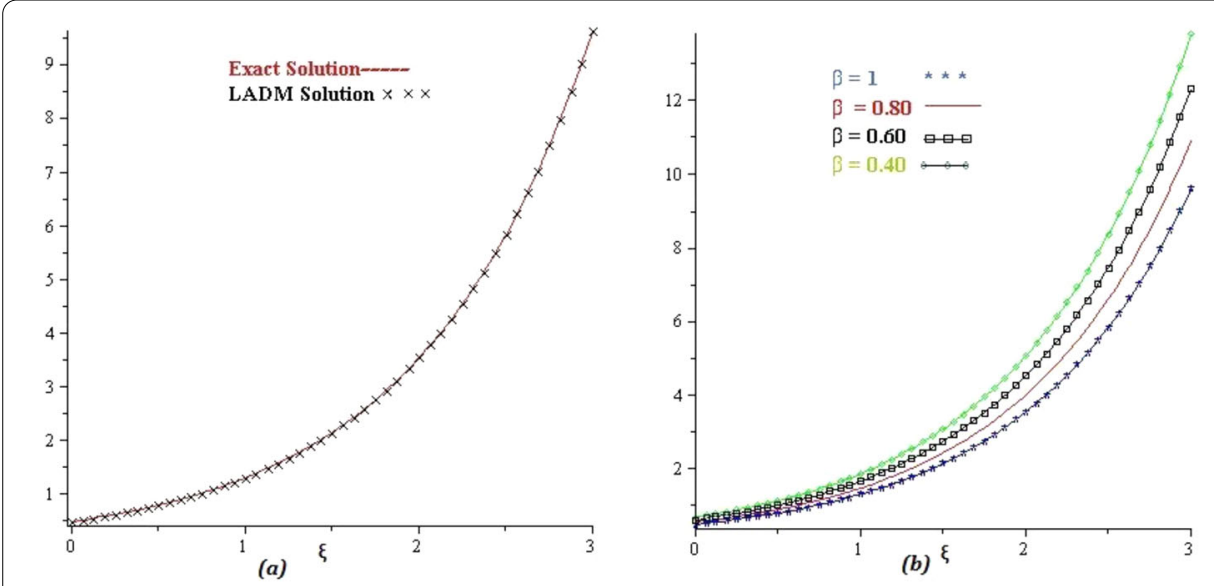

Figure 5 (a) Exact and (b) LADM solution of $u$ for different values of $\beta$ of Example 2
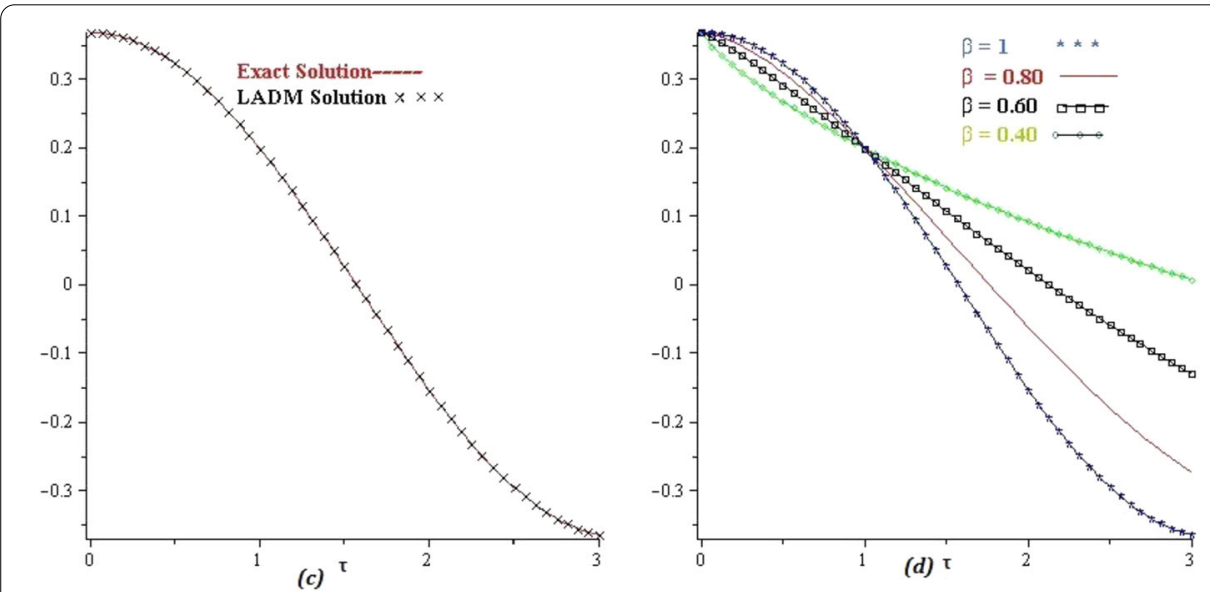

Figure 6 (c) Exact and (d) LADM solution of $v$ for different values of $\beta$ of Example 2

The subsequent terms are

$$
\begin{aligned}
u_{2}(\xi, \tau) & =\mathcal{L}^{-1}\left[\frac{1}{s^{\beta}} \mathcal{L}\left\{v_{0} \frac{\partial u_{1}}{\partial \tau}+v_{1} \frac{\partial u_{0}}{\partial \tau}-u_{0} \frac{\partial v_{1}}{\partial \tau}-u_{1} \frac{\partial v_{0}}{\partial \tau}\right\}\right] \\
& =\sum_{k=0}^{\infty} \frac{\xi^{2 \beta+k}}{\Gamma(2 \beta+k+1)}-\sum_{k=0}^{\infty} \frac{(-\xi)^{2 \beta+k}}{\Gamma(2 \beta+k+1)}-\cos \tau \frac{\xi^{2 \beta}}{\Gamma(2 \beta+1)}, \\
v_{2}(\xi, \tau) & =\mathcal{L}^{-1}\left[\frac{1}{s^{\beta}} \mathcal{L}\left\{-\frac{\partial u_{0}}{\partial \tau} \frac{\partial v_{1}}{\partial \xi}-\frac{\partial u_{1}}{\partial \tau} \frac{\partial v_{0}}{\partial \xi}-\frac{\partial v_{0}}{\partial \tau} \frac{\partial u_{1}}{\partial \xi}-\frac{\partial v_{1}}{\partial \tau} \frac{\partial u_{0}}{\partial \xi}\right\}\right] \\
& =\cos \tau \frac{\xi^{2 \beta-1}}{\Gamma(2 \beta)}+\cos ^{2} \tau \sum_{k=0}^{\infty} \frac{(-\xi)^{2 \beta+k-1}}{\Gamma(2 \beta+k)}+\sin ^{2} \tau \sum_{k=0}^{\infty} \frac{\xi^{2 \beta+k-1}}{\Gamma(2 \beta+k)} .
\end{aligned}
$$

The obtained result for Example 2 is as follows:

$$
\begin{aligned}
& u(\xi, \tau)=u_{0}(\xi, \tau)+u_{1}(\xi, \tau)+u_{2}(\xi, \tau)+u_{3}(\xi, \tau)+\cdots, \\
& v(\xi, \tau)=v_{0}(\xi, \tau)+v_{1}(\xi, \tau)+v_{2}(\xi, \tau)+v_{3}(\xi, \tau)+\cdots,
\end{aligned}
$$




$$
\begin{aligned}
u(\xi, \tau)= & \sin \tau+\sin \tau \xi^{\beta} \sum_{k=0}^{\infty} \frac{\xi^{k}}{\Gamma(k+\beta+1)}+\sum_{k=0}^{\infty} \frac{\xi^{2 \beta+k}}{\Gamma(2 \beta+k+1)} \\
& -\sum_{k=0}^{\infty} \frac{(-\xi)^{2 \beta+k}}{\Gamma(2 \beta+k+1)}-\cos \tau \frac{\xi^{2 \beta}}{\Gamma(2 \beta+1)} \cdots, \\
\nu(\xi, \tau)= & \cos \tau-\frac{\tau^{\beta}}{\Gamma(\beta+1)}-\cos \tau \xi^{\beta} \sum_{k=0}^{\infty} \frac{-\xi^{k}}{\Gamma(k+\beta+1)}+\cos \tau \frac{\xi^{2 \beta-1}}{\Gamma(2 \beta)} \\
& +\cos ^{2} \tau \sum_{k=0}^{\infty} \frac{(-\xi)^{2 \beta+k-1}}{\Gamma(2 \beta+k)}+\sin ^{2} \tau \sum_{k=0}^{\infty} \frac{\xi^{2 \beta+k-1}}{\Gamma(2 \beta+k)}+\cdots,
\end{aligned}
$$

When $\beta=1$, then LADM solution is

$$
\begin{aligned}
& u(\xi, \tau)=e^{\xi} \sin \tau, \\
& v(\xi, \tau)=e^{-\xi} \cos \tau .
\end{aligned}
$$

Example 3 The system of inhomogeneous fractional-order nonlinear PDEs in [40]

$$
\begin{aligned}
& \frac{\partial^{\beta} u}{\partial \tau^{\beta}}-\frac{\partial w}{\partial \xi} \frac{\partial v}{\partial \tau}-\frac{1}{2} \frac{\partial w}{\partial \tau} \frac{\partial^{2} u}{\partial \xi^{2}}=-4 \xi \tau, \\
& \frac{\partial^{\beta} v}{\partial \tau^{\beta}}-\frac{\partial w}{\partial \tau} \frac{\partial^{2} u}{\partial \xi^{2}}=6 \tau \\
& \frac{\partial^{\beta} w}{\partial \tau^{\beta}}-\frac{\partial^{2} u}{\partial \xi^{2}}-\frac{\partial v}{\partial \xi} \frac{\partial w}{\partial \tau}=4 \xi \tau-2 \tau-2, \quad 0<\beta \leq 1
\end{aligned}
$$

with the initial conditions

$$
u(\xi, 0)=\xi^{2}+1, \quad v(\xi, 0)=\xi^{2}-1, \quad w(\xi, 0)=\xi^{2}-1
$$

Taking the Laplace transform of (22), we get

$$
\begin{aligned}
& \mathcal{L}\left[\frac{\partial^{\beta} u}{\partial \tau^{\beta}}\right]=\mathcal{L}\left[\frac{\partial w}{\partial \xi} \frac{\partial v}{\partial \tau}+\frac{1}{2} \frac{\partial w}{\partial \tau} \frac{\partial^{2} u}{\partial \xi^{2}}-4 \xi \tau\right], \\
& \mathcal{L}\left[\frac{\partial^{\beta} v}{\partial \tau^{\beta}}\right]=\mathcal{L}\left[\frac{\partial w}{\partial \tau} \frac{\partial^{2} u}{\partial \xi^{2}}+6 \tau\right], \\
& \mathcal{L}\left[\frac{\partial^{\beta} w}{\partial \tau^{\beta}}\right]=\mathcal{L}\left[\frac{\partial^{2} u}{\partial \xi^{2}}+\frac{\partial v}{\partial \xi} \frac{\partial w}{\partial \tau}+4 \xi \tau-2 \tau-2\right], \\
& s^{\beta} \mathcal{L}[u(\xi, \tau)]-s^{\beta-1}[u(\xi, 0)]=\mathcal{L}\left[\frac{\partial w}{\partial \xi} \frac{\partial v}{\partial \tau}+\frac{1}{2} \frac{\partial w}{\partial \tau} \frac{\partial^{2} u}{\partial \xi^{2}}-4 \xi \tau\right] \\
& s^{\beta} \mathcal{L}[v(\xi, \tau)]-s^{\beta-1}[v(\xi, 0)]=\mathcal{L}\left[\frac{\partial w}{\partial \tau} \frac{\partial^{2} u}{\partial \xi^{2}}+6 \tau\right] \\
& s^{\beta} \mathcal{L}[w(\xi, \tau)]-s^{\beta-1}[w(\xi, 0)]=\mathcal{L}\left[\frac{\partial^{2} u}{\partial \xi^{2}}+\frac{\partial v}{\partial \xi} \frac{\partial w}{\partial \tau}+4 \xi \tau-2 \tau-2\right] .
\end{aligned}
$$

Using the inverse transformation, we have

$$
u(\xi, \tau)=\mathcal{L}^{-1}\left[\frac{u(\xi, 0)}{s}+\frac{1}{s^{\beta}} \mathcal{L}\left[\frac{\partial w}{\partial \xi} \frac{\partial v}{\partial \tau}+\frac{1}{2} \frac{\partial w}{\partial \tau} \frac{\partial^{2} u}{\partial \xi^{2}}-4 \xi \tau\right],\right.
$$




$$
\begin{aligned}
& \nu(\xi, \tau)=\mathcal{L}^{-1}\left[\frac{v(\xi, 0)}{s}+\frac{1}{s^{\beta}} \mathcal{L}\left[\frac{\partial w}{\partial \tau} \frac{\partial^{2} u}{\partial \xi^{2}}+6 \tau\right]\right], \\
& w(\xi, \tau)=\mathcal{L}^{-1}\left[\frac{w(\xi, 0)}{s}+\frac{1}{s^{\beta}} \mathcal{L}\left[\frac{\partial^{2} u}{\partial \xi^{2}}+\frac{\partial v}{\partial \xi} \frac{\partial w}{\partial \tau}+4 \xi \tau-2 \tau-2\right]\right] .
\end{aligned}
$$

Using the ADM procedure, we get

$$
\begin{aligned}
& u_{0}(\xi, \tau)=\mathcal{L}^{-1}\left[\frac{u(\xi, 0)}{s}\right]=\mathcal{L}^{-1}\left[\frac{\xi^{2}+1}{s}\right]=\xi^{2}+1, \\
& v_{0}(\xi, \tau)=\mathcal{L}^{-1}\left[\frac{v(\xi, 0)}{s}\right]=\mathcal{L}^{-1}\left[\frac{\xi^{2}-1}{s}\right]=\xi^{2}-1, \\
& w_{0}(\xi, \tau)=\mathcal{L}^{-1}\left[\frac{w(\xi, 0)}{s}\right]=\mathcal{L}^{-1}\left[\frac{\xi^{2}-1}{s}\right]=\xi^{2}-1, \\
& u_{j+1}(\xi, \tau)=\mathcal{L}^{-1}\left[\frac{1}{s^{\beta}} \mathcal{L}\left[\frac{\partial w_{j}}{\partial \xi} \frac{\partial v_{j}}{\partial \tau}+\frac{1}{2} \frac{\partial w_{j}}{\partial \tau} \frac{\partial^{2} u_{j}}{\partial \xi^{2}}-4 \xi \tau\right]\right], \\
& v_{j+1}(\xi, \tau)=\mathcal{L}^{-1}\left[\frac{1}{s^{\beta}} \mathcal{L}\left[\frac{\partial w_{j}}{\partial \tau} \frac{\partial^{2} u_{j}}{\partial \xi^{2}}+6 \tau\right]\right], \\
& w_{j+1}(\xi, \tau)=\mathcal{L}^{-1}\left[\frac{1}{s^{\beta}} \mathcal{L}\left[\frac{\partial^{2} u_{j}}{\partial \xi^{2}}+\frac{\partial v_{j}}{\partial \xi} \frac{\partial w_{j}}{\partial \tau}+4 \xi \tau-2 \tau-2\right]\right], \\
& j=0,1,2, \ldots
\end{aligned}
$$

for $j=0$

$$
\begin{aligned}
& u_{1}(\xi, \tau)=\mathcal{L}^{-1}\left[\frac{1}{s^{\beta}} \mathcal{L}\left[\frac{\partial w_{0}}{\partial \xi} \frac{\partial v_{0}}{\partial \tau}+\frac{1}{2} \frac{\partial w_{0}}{\partial \tau} \frac{\partial^{2} u_{0}}{\partial \xi^{2}}-4 \xi \tau\right]\right], \\
& u_{1}(\xi, \tau)=\mathcal{L}^{-1}\left[\frac{-4 \xi}{s^{\beta+2}}\right]=\frac{-4 \xi \tau^{\beta+1}}{\Gamma(\beta+2)}, \\
& v_{1}(\xi, \tau)=\mathcal{L}^{-1}\left[\frac{1}{s^{\beta}} \mathcal{L}\left[\frac{\partial w_{0}}{\partial \tau} \frac{\partial^{2} u_{0}}{\partial \xi^{2}}+6 \tau\right]\right], \\
& v_{1}(\xi, \tau)=\mathcal{L}^{-1}\left[\frac{6}{s^{\beta+2}}\right]=\frac{6 \tau^{\beta+1}}{\Gamma(\beta+2)}, \\
& w_{1}(\xi, \tau)=\mathcal{L}^{-1}\left[\frac{1}{s^{\beta}} \mathcal{L}\left[\frac{\partial^{2} u_{0}}{\partial \xi^{2}}+\frac{\partial v_{0}}{\partial \xi} \frac{\partial w_{0}}{\partial \tau}+4 \xi \tau-2 \tau-2\right]\right], \\
& w_{1}(\xi, \tau)=\mathcal{L}^{-1}\left[\frac{4 \xi}{s^{\beta+2}}-\frac{2}{s^{\beta+2}}\right]=\frac{4 \xi \tau^{\beta+1}}{\Gamma(\beta+2)}-\frac{2 \tau^{\beta+1}}{\Gamma(\beta+2)} .
\end{aligned}
$$

The subsequent terms are

$$
\begin{aligned}
u_{2}(\xi, \tau) & =\mathcal{L}^{-1}\left[\frac{1}{s^{\beta}} \mathcal{L}\left[\frac{\partial w_{0}}{\partial \xi} \frac{\partial v_{1}}{\partial \tau}+\frac{\partial w_{1}}{\partial \xi} \frac{\partial v_{0}}{\partial \tau}+\frac{1}{2} \frac{\partial w_{0}}{\partial \tau} \frac{\partial^{2} u_{1}}{\partial \xi^{2}}+\frac{1}{2} \frac{\partial w_{1}}{\partial \tau} \frac{\partial^{2} u_{0}}{\partial \xi^{2}}\right]\right] \\
& =\frac{16 \xi \tau^{2 \beta}}{\Gamma(\beta+2)}-\frac{2 \tau^{\beta}}{\Gamma(\beta+2)}
\end{aligned}
$$




$$
\begin{aligned}
& \nu_{2}(\xi, \tau)=\mathcal{L}^{-1}\left[\frac{1}{s^{\beta}} \mathcal{L}\left[\frac{\partial w_{0}}{\partial \tau} \frac{\partial^{2} u_{1}}{\partial \xi^{2}}+\frac{\partial w_{1}}{\partial \tau} \frac{\partial^{2} u_{0}}{\partial \xi^{2}}\right]\right], \\
& =\frac{8 \xi \tau^{2 \beta}}{\Gamma(\beta+2)}-\frac{4 \tau^{2 \beta}}{\Gamma(\beta+2)}, \\
& w_{2}(\xi, \tau)=\mathcal{L}^{-1}\left[\frac{1}{s^{\beta}} \mathcal{L}\left[\frac{\partial^{2} u_{1}}{\partial \xi^{2}}+\frac{\partial v_{0}}{\partial \xi} \frac{\partial w_{1}}{\partial \tau}+\frac{\partial v_{1}}{\partial \xi} \frac{\partial w_{0}}{\partial \tau}\right]\right], \\
& =\frac{8 \xi^{2} \tau^{2 \beta}}{\Gamma(\beta+2)}-\frac{4 \xi \tau^{2 \beta}}{\Gamma(\beta+2)} \text {, } \\
& u_{3}(\xi, \tau)=\mathcal{L}^{-1}\left[\frac{1}{s^{\beta}} \mathcal{L}\left[\frac{\partial w_{0}}{\partial \xi} \frac{\partial v_{2}}{\partial \tau}+\frac{\partial w_{1}}{\partial \xi} \frac{\partial v_{1}}{\partial \tau}+\frac{\partial w_{2}}{\partial \xi} \frac{\partial v_{0}}{\partial \tau}\right]\right] \\
& +\mathcal{L}^{-1}\left[\frac{1}{s^{\beta}} \mathcal{L}\left[\frac{1}{2} \frac{\partial w_{0}}{\partial \tau} \frac{\partial^{2} u_{2}}{\partial \xi^{2}}+\frac{1}{2} \frac{\partial w_{1}}{\partial \tau} \frac{\partial^{2} u_{1}}{\partial \xi^{2}}+\frac{1}{2} \frac{\partial w_{2}}{\partial \tau} \frac{\partial^{2} u_{0}}{\partial \xi^{2}}\right]\right], \\
& =\left(\frac{4(\beta+1) \Gamma(2 \beta+2)}{\Gamma(\beta+2) \Gamma(\beta+2) \Gamma(3 \beta+2)}\right) \tau^{3 \beta+1}+\left(\frac{48 \xi^{2}(\beta) \Gamma(2 \beta)}{\Gamma(\beta+2) \Gamma(3 \beta)}\right) \tau^{3 \beta-1} \\
& -\left(\frac{24 \xi(\beta) \Gamma(2 \beta)}{\Gamma(\beta+2) \Gamma(3 \beta)}\right) \tau^{3 \beta-1}, \\
& v_{3}(\xi, \tau)=\mathcal{L}^{-1}\left[\frac{1}{s^{\beta}} \mathcal{L}\left[\frac{\partial w_{0}}{\partial \tau} \frac{\partial^{2} u_{2}}{\partial \xi^{2}}+\frac{\partial w_{1}}{\partial \tau} \frac{\partial^{2} u_{1}}{\partial \xi^{2}}+\frac{\partial w_{2}}{\partial \tau} \frac{\partial^{2} u_{0}}{\partial \xi^{2}}\right]\right], \\
& =\left(\frac{32 \xi^{2} \beta \Gamma(2 \beta)}{\Gamma(\beta+2) \Gamma(3 \beta)}\right) \tau^{3 \beta-1}-\left(\frac{16 \xi \beta \Gamma(2 \beta)}{\Gamma(\beta+2) \Gamma(3 \beta)}\right) \tau^{3 \beta-1}, \\
& w_{3}(\xi, \tau)=\mathcal{L}^{-1}\left[\frac{1}{s^{\beta}} \mathcal{L}\left[\frac{\partial^{2} u_{2}}{\partial \xi^{2}}+\frac{\partial v_{0}}{\partial \xi} \frac{\partial w_{2}}{\partial \tau}+\frac{\partial v_{1}}{\partial \xi} \frac{\partial w_{1}}{\partial \tau}+\frac{\partial v_{2}}{\partial \xi} \frac{\partial w_{0}}{\partial \tau}\right]\right], \\
& =\left(\frac{32 \xi^{3} \beta \Gamma(2 \beta)}{\Gamma(\beta+2) \Gamma(3 \beta)}\right) \tau^{3 \beta-1}-\left(\frac{16 \xi^{2} \beta \Gamma(2 \beta)}{\Gamma(\beta+2) \Gamma(3 \beta)}\right) \tau^{3 \beta-1} \text {. }
\end{aligned}
$$

The obtained result for Example 3

$$
\begin{aligned}
u(\xi, \tau)= & u_{0}(\xi, \tau)+u_{1}(\xi, \tau)+u_{2}(\xi, \tau)+u_{3}(\xi, \tau)+\cdots \\
v(\xi, \tau)= & v_{0}(\xi, \tau)+v_{1}(\xi, \tau)+v_{2}(\xi, \tau)+v_{3}(\xi, \tau)+\cdots \\
w(\xi, \tau)= & w_{0}(\xi, \tau)+w_{1}(\xi, \tau)+w_{2}(\xi, \tau)+w_{3}(\xi, \tau)+\cdots, \\
u(\xi, \tau)= & \xi^{2}+1-\frac{4 \xi \tau^{\beta+1}}{\Gamma(\beta+2)}+\frac{16 \xi \tau^{2 \beta}}{\Gamma(\beta+2)}-\frac{2 \tau^{\beta}}{\Gamma(\beta+2)} \\
& +\left(\frac{4(\beta+1) \Gamma(2 \beta+2)}{\Gamma(\beta+2) \Gamma(\beta+2) \Gamma(3 \beta+2)}\right) \tau^{3 \beta+1} \\
& +\left(\frac{48 \xi^{2}(\beta) \Gamma(2 \beta)}{\Gamma(\beta+2) \Gamma(3 \beta)}\right) \tau^{3 \beta-1} \\
& -\left(\frac{24 \xi(\beta) \Gamma(2 \beta)}{\Gamma(\beta+2) \Gamma(3 \beta)}\right) \tau^{3 \beta-1}+\cdots, \\
v(\xi, \tau)= & \xi^{2}-1+\frac{6 \tau^{\beta+1}}{\Gamma(\beta+2)}+\frac{8 \xi \tau^{2 \beta}}{\Gamma(\beta+2)}-\frac{4 \tau^{2 \beta}}{\Gamma(\beta+2)} \\
& +\left(\frac{32 \xi^{2} \beta \Gamma(2 \beta)}{\Gamma(\beta+2) \Gamma(3 \beta)}\right) \tau^{3 \beta-1}-\left(\frac{16 \xi \beta \Gamma(2 \beta)}{\Gamma(\beta+2) \Gamma(3 \beta)}\right) \tau^{3 \beta-1}+\cdots
\end{aligned}
$$



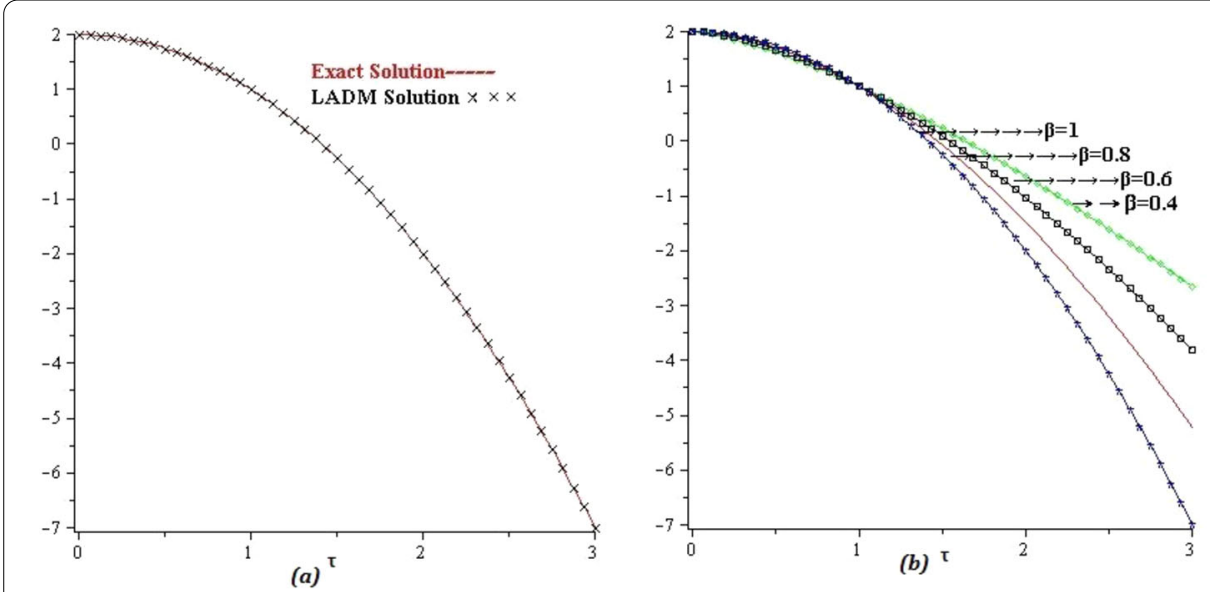

Figure 7 (a) Exact and (b) LADM solution for Example 3 of $u$ at different values of $\beta$
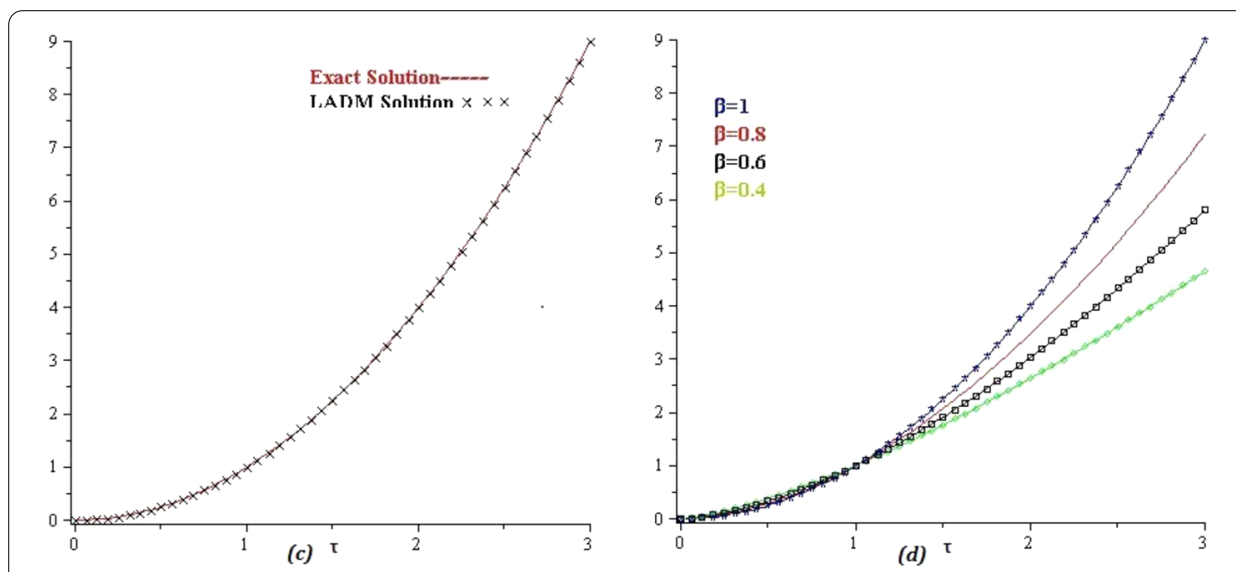

Figure 8 (c) Exact and (d) LADM solution for Example 3 of $v$ at different values of $\beta$

$$
\begin{aligned}
w(\xi, \tau)= & \xi^{2}-1+\frac{4 \xi \tau^{\beta+1}}{\Gamma(\beta+2)}-\frac{2 \tau^{\beta+1}}{\Gamma(\beta+2)}+\frac{8 \xi^{2} \tau^{2 \beta}}{\Gamma(\beta+2)}-\frac{4 \xi \tau^{2 \beta}}{\Gamma(\beta+2)} \\
& +\left(\frac{32 \xi^{3} \beta \Gamma(2 \beta)}{\Gamma(\beta+2) \Gamma(3 \beta)}\right) \tau^{3 \beta-1}-\left(\frac{16 \xi^{2} \beta \Gamma(2 \beta)}{\Gamma(\beta+2) \Gamma(3 \beta)}\right) \tau^{3 \beta-1}+\cdots
\end{aligned}
$$

When $\beta=1$, then LADM solution is

$$
\begin{gathered}
u(\xi, \tau)=\xi^{2}-\tau^{2}+1, \\
v(\xi, \tau)=\xi^{2}+\tau^{2}-1, \\
w(\xi, \tau)=\xi^{2}-\tau^{2}-1 .
\end{gathered}
$$

\section{Conclusion}

In this research paper, a powerful analytical technique, called LADM, is applied to find the solution of some important system of fractional-order partial differential equations. The obtained results are interesting and also in good agreement towards the exact solutions. 

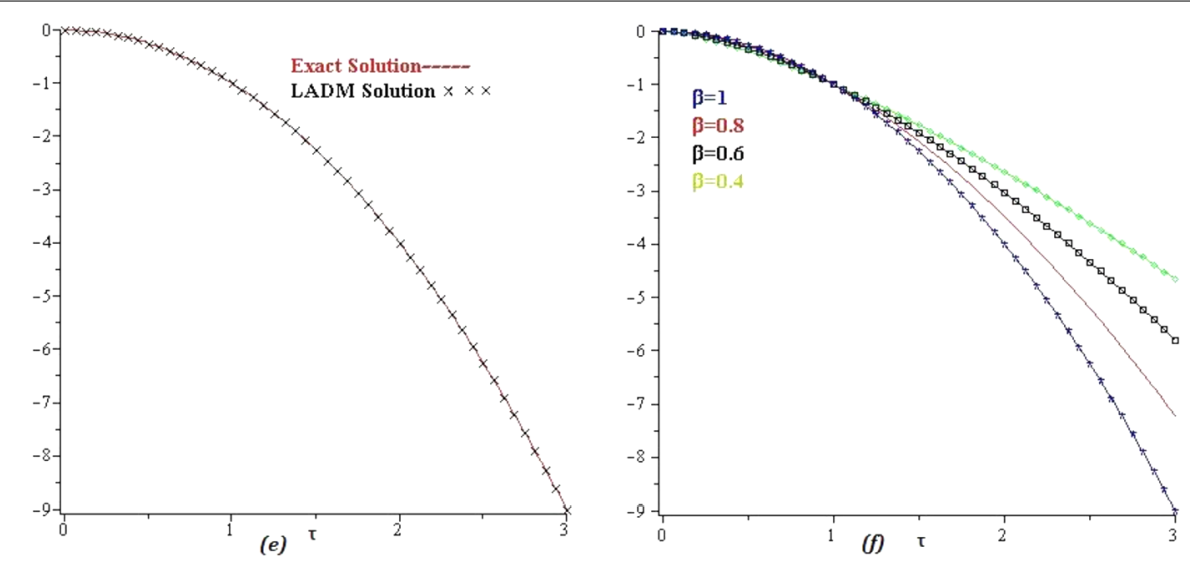

Figure 9 (e) Exact and (f) LADM solution for Example 3 of $w$ at different values of $\beta$

The behavior and validity of the present method is checked by taking some numerical examples. The procedure and results of LADM have shown higher accuracy of the current method as compared to other methods in literature. The convergence of fractionalorder solutions towards integer-order solution can be observed from the graphs in the paper.

\section{Acknowledgements}

The authors thank the editor and anonymous reviewers for their valuable suggestions which substantially improved the quality of the paper.

\section{Funding}

Theoretical and Computational Science (TaCS) Center Department of Mathematics, Faculty of Science, King Mongkuts University of Technology Thonburi (KMUTT). 126 Pracha Uthit Rd., Bang Mod, Thung Khru, 10140,Bangkok, Thailand.

Availability of data and materials

Not applicable.

\section{Competing interests}

The authors declare that there is no conflict of interests regarding the publication of this article.

\section{Authors' contributions}

The authors declare that this study was accomplished in collaboration with the same responsibility. All authors read and approved the final manuscript.

\section{Author details}

'Department of Mathematics, Abdul Wali khan University, 23200 Mardan, Pakistan. ${ }^{2}$ Theoretical and Computational Science (TaCS) Center Department of Mathematics, Faculty of Science, King Mongkuts University of Technology Thonburi (KMUTT), 126 Pracha Uthit Rd., Bang Mod, Thung Khru, 10140 Bangkok, Thailand. ${ }^{3}$ Department of Medical Research, China Medical University Hospital, China Medical University, 40402 Taichung, Taiwan. ${ }^{4}$ Department of Mathematics, Faculty of Arts and Sciences, Cankaya University, 06530 Ankara, Turkey. ${ }^{5}$ Institute of Space Sciences, Magurele-Bucharest, Romania.

\section{Publisher's Note}

Springer Nature remains neutral with regard to jurisdictional claims in published maps and institutional affiliations.

Received: 3 January 2020 Accepted: 14 July 2020 Published online: 22 July 2020

\section{References}

1. Javeed, S., Saif, S., Waheed, A., Baleanu, D.: Exact solutions of fractional mBBM equation and coupled system of fractional Boussinesq-Burgers. Results Phys. 9, 1275-1281 (2018)

2. Abassy, T.A., El-Tawil, M.A., El-Zoheiry, H.: Modified variational iteration method for Boussinesq equation. Comput. Math. Appl. 54(7-8), 955-965 (2007)

3. Jothimani, K., Kaliraj, K., Hammouch, Z., Ravichandran, C.: New results on controllability in the framework of fractional integrodifferential equations with nondense domain. Eur. Phys. J. Plus 134(9), 441 (2019) 
4. Ait Touchent, K., Hammouch, Z., Mekkaoui, T., Belgacem, F.: Implementation and convergence analysis of homotopy perturbation coupled with Sumudu transform to construct solutions of local-fractional PDEs. Fractal Fract. 2(3), 22 (2018)

5. Ansari, F.A., Ahmed, I., Dharejo, K.A., Kalhoro, A.N.: Computational and analytical solution of fractional order linear partial differential equations using Sumudu transform and its properties. Int. J. Comput. Sci. Netw. Secur. 18(9), 43-50 (2018)

6. Shah, R., Farooq, U., Khan, H., Baleanu, D., Kumam, P., Arif, M.: Fractional view analysis of third order Kortewege-De Vries equations, using a new analytical technique. Front. Phys. 7, 244 (2020). https://doi.org/10.3389/fphy

7. Ganji, R.M., Jafari, H.: A new approach for solving nonlinear Volterra integro-differential equations with Mittag-Leffler kernel. Proc. Inst. Math. Mech. Natl. Acad. Sci. Azerb. 46(1), 144-158 (2020)

8. Shah, R., Khan, H., Baleanu, D., Kumam, P., Arif, M.: A semi-analytical method to solve family of Kuramoto-Sivashinsky equations. J. Taibah Univ. Sci. 14(1), 402-411 (2020)

9. Shah, R., Khan, H., Baleanu, D., Kumam, P., Arif, M.: A novel method for the analytical solution of fractional Zakharov-Kuznetsov equations. Adv. Differ. Equ. 2019(1), 1 (2019)

10. Kadkhoda, N., Jafari, H.: An analytical approach to obtain exact solutions of some space-time conformable fractional differential equations. Adv. Differ. Equ. 2019(1), 428 (2019)

11. Babaei, A., Jafari, H., Ahmadi, M.: A fractional order HIV/AIDS model based on the effect of screening of unaware infectives. Math. Methods Appl. Sci. 42(7), 2334-2343 (2019)

12. Mohammed, O.H.: Solving parabolic partial differential equations using modified Bellman's method with G-spline interpolation. Baghdad Sci. J. 7(4), 1447-1454 (2010)

13. Khan, H., Shah, R., Baleanu, D., Kumam, P., Arif, M.: Analytical solution of fractional-order hyperbolic telegraph equation, using natural transform decomposition method. Electron. 8(9), 1015 (2019)

14. Mohsin, A.K., Mohammed, O.H.: Homotopy analysis and adomian decomposition methods for approximating the solution of two dimensional partial integro-differential equations of fractional order

15. Khan, H., Shah, R., Kumam, P., Arif, M.: Analytical solutions of fractional-order heat and wave equations by the natura transform decomposition method. Entropy 21(6), 597 (2019)

16. Shah, R., Khan, H., Mustafa, S., Kumam, P., Arif, M.: Analytical solutions of fractional-order diffusion equations by natural transform decomposition method. Entropy 21(6), 557 (2019)

17. Mohammed, O.H., Wadi, Q.: A modified method for solving delay differential equations of fractional order. IOSR J. Math. 12(3), 15-21 (2016)

18. Darzi, R., Agheli, B.: Analytical approach to solving fractional partial differential equation by optimal q-homotopy analysis method. Numer. Anal. Appl. 11(2), 134-145 (2018)

19. Al-Sabbagh, A.A., Hanan, I.K., Mohammed, O.H.: Some numerical methods for solving fractional parabolic partial differential equations. Eng. Technol. J. 28(12), 2480-2485 (2010)

20. Khan, Y., Vazquez-Leal, H., Faraz, N.: An auxiliary parameter method using Adomian polynomials and Laplace transformation for nonlinear differential equations. Appl. Math. Model. 37(5), 2702-2708 (2013)

21. Nofal, T.A.: Simple equation method for nonlinear partial differential equations and its applications. J. Egypt. Math Soc. 24(2), 204-209 (2016)

22. Jafari, H., Nazari, M., Baleanu, D., Khalique, C.M.: A new approach for solving a system of fractional partial differential equations. Comput. Math. Appl. 66(5), 838-843 (2013)

23. Sirisubtawee, S., Koonprasert, S.: Exact traveling wave solutions of certain nonlinear partial differential equations using the-expansion method. Adv. Math. Phys. 2018, Article ID 7628651 (2018). https://doi.org/10.1155/2018/7628651

24. Vanani, S.K., Aminataei, A.: Tau approximate solution of fractional partial differential equations. Comput. Math. Appl. 62(3), 1075-1083 (2011)

25. Özpınar, F.: Applying discrete homotopy analysis method for solving fractional partial differential equations. Entropy 20(5), $332(2018)$

26. Shah, R., Khan, H., Kumam, P., Arif, M.: An analytical technique to solve the system of nonlinear fractional partial differential equations. Mathematics 7(6), 505 (2019)

27. Khan, H., Shah, R., Kumam, P., Baleanu, D., Arif, M.: An efficient analytical technique, for the solution of fractional-order telegraph equations. Mathematics 7(5), 426 (2019)

28. Jafari, H., Khalique, C.M., Nazari, M.: Application of the Laplace decomposition method for solving linear and nonlinear fractional diffusion-wave equations. Appl. Math. Lett. 24(11), 1799-1805 (2011)

29. Mohamed, M.Z:: Comparison between the Laplace decomposition method and Adomian decomposition in time-space fractional nonlinear fractional differential equations. Appl. Math. 9(04), 448 (2018)

30. Gaxiola, O.G.: The Laplace-Adomian decomposition method applied to the Kundu-Eckhaus equation. Int. J. Math. Appl. 5(1-a), 1-12 (2017)

31. Al-Zurigat, M.: Solving nonlinear fractional differential equation using a multi-step Laplace Adomian decomposition method. An. Univ. Craiova, Ser. Mat. Inform. 39(2), 200-210 (2012)

32. Mohammed, O.H., Salim, H.A.: Computational methods based Laplace decomposition for solving nonlinear system of fractional order differential equations. Alex. Eng. J. 57(4), 3549-3557 (2018)

33. Haq, F., Shah, K., ur Rahman, G., Shahzad, M.: Numerical solution of fractional order smoking model via Laplace Adomian decomposition method. Alex. Eng. J. 57(2), 1061-1069 (2018)

34. Mahmood, S., Shah, R., Arif, M.: Laplace Adomian decomposition method for multi dimensional time fractional model of Navier-Stokes equation. Symmetry 11(2), 149 (2019)

35. Shah, R., Khan, H., Arif, M., Kumam, P.: Application of Laplace-Adomian decomposition method for the analytical solution of third-order dispersive fractional partial differential equations. Entropy 21(4), 335 (2019)

36. Hilfer, R.: Applications of Fractional Calculus in Physics. World Scientific, River Edge (2000)

37. Miller, K.S., Ross, B.: An introduction to the fractional calculus and fractional differential equations (1993)

38. Podlubny, I.: Fractional Differential Equations: An Introduction to Fractional Derivatives, Fractional Differential Equations, to Methods of Their Solution and Some of Their Applications, vol. 198. Elsevier, Amsterdam (1998)

39. Naghipour, A., Manafian, J.: Application of the Laplace Adomian decomposition and implicit methods for solving Burgers' equation. TWMS J. Pure Appl. Math. 6, 68-77 (2015) 
40. Biazar, J., Eslami, M.: A new homotopy perturbation method for solving systems of partial differential equations. Comput. Math. Appl. 62(1), 225-234 (2011)

Submit your manuscript to a SpringerOpen ${ }^{\circ}$ journal and benefit from:

- Convenient online submission

- Rigorous peer review

- Open access: articles freely available online

- High visibility within the field

- Retaining the copyright to your article

Submit your next manuscript at $\gg$ springeropen.com 